\title{
Paradigms of pattern formation: Towards a computational theory of morphogenesis
}

Przemyslaw Prusinkiewicz

University of Calgary.

\section{Abstract}

The variety of natural patterns makes it difficult to analyze and compare them in a systematic manner. We address this problem by focusing on the computational aspects of pattern formation processes. They are characterized in terms of the number of morphogenetic agents, the computing capability of each agent, and the forms of information transfer between the agents and their environment. This computational analysis can be applied to a wide range of patterns. It highlights the fundamental, algorithmic similarities between processes that may be implemented using different physical or physiological mechanisms in nature. It also confirms earlier observations that similar or identical patterns can be created by fundamentally different processes. The tradeoffs between computational characteristics of these processes lend themselves to a formal analysis, which could lead to the formulation of a "computational theory of morphogenesis" on the basis of the theory of algorithms.

\section{Reference}

Przemyslaw Prusinkiewicz: Paradigms of pattern formation: Towards a computational theory of morphogenesis. In Pattern Formation in Biology, Vision, and Dynamics, pp. 91-95. 


\title{
PARADIGMS OF PATTERN FORMATION: TOWARDS A COMPUTATIONAL THEORY OF MORPHOGENESIS
}

\author{
PRZEMYSLAW PRUSINKIEWICZ \\ Department of Computer Science, The University of Calgary \\ 2500 University Dr. N.W., Calgary, Alberta T2N 1N4, Canada \\ E-mail: pwp@cpsc.ucalgary.ca
}

\begin{abstract}
The variety of natural patterns makes it difficult to analyze and compare them in a systematic manner. We address this problem by focusing on the computational aspects of pattern formation processes. They are characterized in terms of the number of morphogenetic agents, the computing capability of each agent, and the forms of information transfer between the agents and their environment. This computational analysis can be applied to a wide range of patterns. It highlights the fundamental, algorithmic similarities between processes that may be implemented using different physical or physiological mechanisms in nature. It also confirms earlier observations that similar or identical patterns can be created by fundamentally different processes. The tradeoffs between computational characteristics of these processes lend themselves to a formal analysis, which could lead to the formulation of a "computational theory of morphogenesis" on the basis of the theory of algorithms.
\end{abstract}

\section{Introduction}

What is a pattern? In their treatise Tilings and Patterns ${ }^{7}$, Grünbaum and Shephard wrote: "There seems to be not a single instance in the literature of a meaningful definition of a 'pattern' that is, in any sense, useful" (page 261). To address that situation, they introduced the notion of discrete patterns, which is based on repetitive occurrences of predefined figures called motifs. A pattern is assumed to be invariant with respect to some isometries in the plane, called its symmetries. The set of all symmetries forms a group under composition. ${ }^{7}$

The precision of this definition also makes it narrow: it does not encompass many geometric objects that, intuitively, we consider as patterns. This limitation was clearly stated in a subsequent paper by Grünbaum: ${ }^{6}$

Sociologists of mathematics should have a field day investigating the mob phenomena that are apparent in symmetry analysis of repeating patterns. What started as a suggestion by Pólya ${ }^{24}$ - that one could study ornaments from the point of view of the discrete symmetry groups - soon turned [...] into a dogma. [...] If just a small fraction of the energies that go into trying to force patterns and phenomena into a group-theoretic mold were devoted to the development of other approaches more suited in specific circumstances, both mathematics and its applications would profit.

What conceptual frameworks could be thus used to describe and analyze patterns that lack the strict repetitiveness dictated by the symmetry groups? Such patterns abound in the natural world and include, to name but a few examples for which mathematical models have been proposed, branching patterns of plants and rivers, venation patterns in leaves, arrangements of cells in organs of plants and animals, 

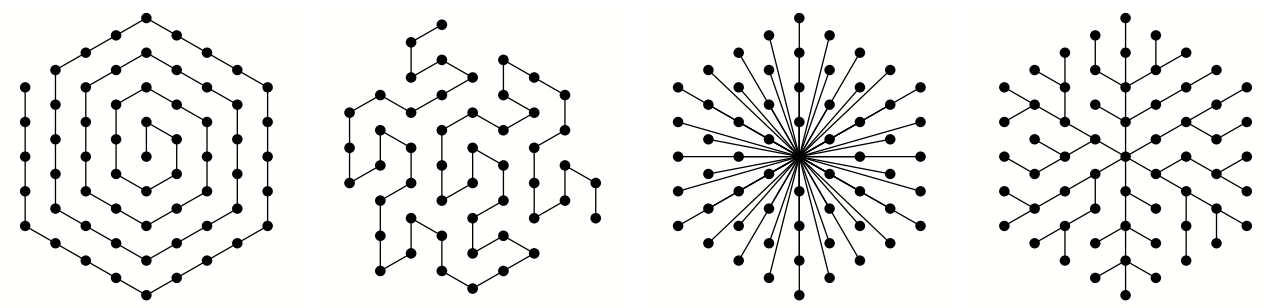

Figure 1: Prototypical patterns identified by Stevens: ${ }^{38}$ a spiral, a meander, an explosion, and a branching pattern

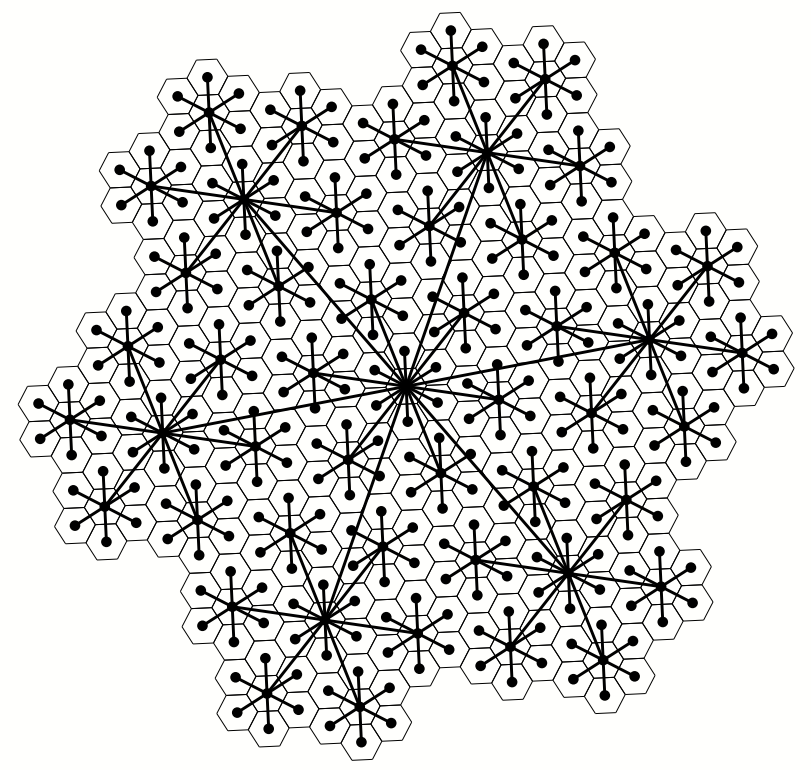

Figure 2: A variant of the branching pattern

pigmentation patterns in sea shells and butterfly wings, and animal coat patterns (see ${ }^{26}$ for a review). Is it possible to look at these patterns in some systematic, unified manner? Can we compare different patterns, the natural mechanisms that brought them into being, and their mathematical models, using a consistent set of uniform criteria?

At some levels of abstraction, these questions have been considered in the past. Stevens ${ }^{38}$ distinguished four prototypical classes of patterns — spirals, meanders, explosions, and branching patterns - by considering different methods for connecting a set of regularly arranged points into a graph without cycles (Figure 1). The definition of each class allows for some degree of variation, with the most significant differences occurring among branching patterns. For example, Figure 2 shows a combination of a branching pattern and an explosion. 
Patterns in each class were characterized by geometric attributes: uniformity, space filling, overall length of lines, and directness of lines. This characterization proved useful when analyzing natural patterns from the viewpoint of their optimality. Such analysis has a long tradition in the description of natural patterns and forms. ${ }^{9,41}$ Stevens extended this analysis to human-made forms, and noted that: ${ }^{38}$

In the distribution of blood vessels, the route of a subway system, or the design of forked columns to carry a roof where both overall length and directness are important, the same branching pattern might turn out to be advantageous.

Unfortunately, the optimality of the result does not offer a direct insight into the mechanisms that govern pattern formation. A classification that addressed this limitation was suggested by Bell, who distinguished the following three categories of branching patterns: ${ }^{3}$

- 'Blind' patterns, in which branch initiation is controlled solely by the imposed programme rules, that is from without the 'organism' and its 'environment'.

- 'Sighted' patterns, in which the initiation of a new branch is influenced by factors detected by it in the immediate neighborhood, such as proximity of other organisms, or parts of the same organism.

- Self-regulatory patterns, in which branch initiation is controlled by the developing simulation itself, using communication via components of the existing framework, whether or not affected by 'environmental' factors.

This categorization is appealing for several reasons:

- It focuses on the processes of pattern formation, rather than patterns themselves. This is consistent with the biological tradition of viewing patterns and forms as derivative of developmental processes. In the words of d'Arcy Thompson, "growth deserves to be studied as a necessary preliminary to the theoretical study of form. "41

- It sets the stage for addressing questions regarding causal relationships between the local rules and the resulting global patterns. These questions are essential to our understanding of nature, since local interactions can be implemented by a wide range of physical, chemical, physiological, and even cognitive processes.

- It focuses on the fundamental, algorithmic properties of pattern formation. This makes it possible to recognize and analyze similarities between apparently different realizations of similar patterns, such as the branching structures of plants and the branching foraging patterns of ants. ${ }^{2,10}$

- It does not presuppose any computational framework for model construction, such as cellular automata, ${ }^{42}$ L-systems, ${ }^{15,31}$ or reaction-diffusion models. ${ }^{20, ?}$ Consequently, analysis of the algorithmic nature of pattern formation processes may remain unobscured by the technical aspects of model implementations. 


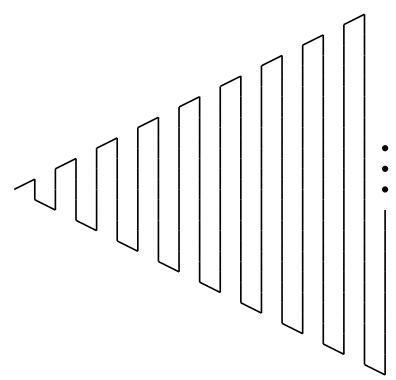

Figure 3: A zig-zag pattern

Many authors have observed that different algorithms may produce similar or identical patterns. The most extensive illustration of this point was given by Held, who devoted an entire book to the discussion of various processes that produce a pattern of periodic stripes. ${ }^{8}$ Other case studies of different algorithms that may produce similar patterns deal with the control of branching in trees ${ }^{11}$ and the flowering sequences in compound inflorescences. ${ }^{31}$

Since there is not a one-to-one correspondence between the patterns and the mechanisms that generate them, one can ask questions about the relationships between different processes that produce identical or similar patterns. In this paper we consider these questions in the context of Bell's caterization of pattern formation processes. Using a simple pattern as an example — a zig-zag with a growing amplitude (Figure 3) — we show that differences between generation mechanisms involve tradeoffs in features: the number of pattern-formation agents, the synchronization of their operation, the amount of computation that they carry out, the amount of information that they can memorize, the type of information that is exchanged between the agents, the structure and the environment, and the paths of information flow.

\section{Single blind agent with finite memory}

Let us consider the zig-zag pattern shown in Figure 3 as the path of an agent a turtle $^{1}$ — that traces a line as it moves in the plane. The turtle operates in discrete steps: and in each step it draws a line segment of a given length or turns left or right by a given angle. The performed action is decided by a control system in the turtle's "head". Clearly, the class of patterns that can be traced by the turtle depends on the computational power of its control system. In this section we consider a "blind turtle with finite memory", in which the control is effected by an autonomous deterministic finite automaton (autonomous DFA). These terms mean that: (i) the turtle has finite memory, represented by a finite number of discrete states; (ii) in each step, the next state is specified in a deterministic fashion; (iii) the next state depends only on the previous state and, in particular, is not affected by any external input; and (iv) the action performed by the turtle in each step is determined by its state, thus the number of possible actions is finite as well. A 

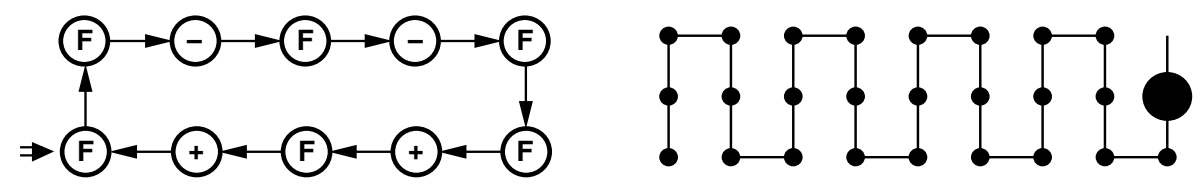

Figure 4: An autonomous finite automaton controlling the sequence of a turtle's movements (left), and the resulting pattern (right)

\section{ப்பாள்பா}

Figure 5: A frieze pattern generated by an autonomous finite automaton controlling a turtle

sample autonomous DFA is defined by the graph shown on the left side of Figure 4. Circles represent states, with the double arrow pointing to the initial state. Symbols in the circles indicate the output that controls the turtle: move forward and draw a line of unit length $(F)$, turn to the left by $90^{\circ}(+)$, and turn to the right by $90^{\circ}(-)$. Arrows define the state transition function, which determines the next state of the automaton given its current state. As the automaton cycles through the sequence of line-drawing steps and turns, the turtle creates the zig-zag of constant amplitude shown on the right side of Figure 4. The dots in this pattern indicate its partition into individual line segments. The larger black circle represents the turtle. The short line segment associated with the turtle indicates its orientation.

A control automaton with more states may yield the frieze pattern ${ }^{18}$ shown in Figure 5. Yet the question remains whether we can define an autonomous turtle with finite control that would trace the (infinite) zig-zag curve of growing amplitude, shown in Figure 3. This turns out to be impossible. As each state of an autonomous FDA determines the next state uniquely, the automaton must settle into a fixed cycle of state transitions after a finite number of non-periodic steps at most. Thus, any figure traced by a turtle with finite memory must have an ultimately periodic character. The zig-zag pattern of Figure 3 is not periodic, because its corners are separated by increasingly long vertical lines. Consequently, it cannot be generated by a turtle with an autonomous finite-state control.

In spite of its simplicity, the autonomous FDA pattern formation model yields many questions. For example, what are the properties of paths created by a blind finite-memory turtle on a sphere or on a torus? What would happen if the blind turtle was moving in an expanding space? What would be the consequences of allowing a turtle to create new turtles, which would contribute lines to the overall pattern on their own? Some of these questions will be addressed in Section 7.

\section{Single blind agent with infinite memory}

How can we augment the capabilities of the turtle so that it can create a zig-zag of increasing amplitude? One intuitively obvious possibility is to allow the turtle to count. In the case illustrated in Figure 6, the turtle is equipped with two counters, 

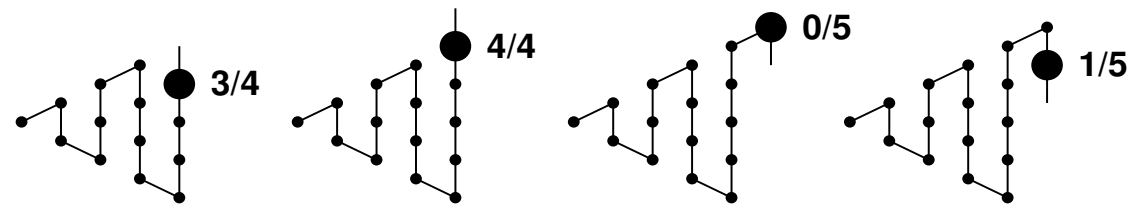

Figure 6: Four consecutive steps in the creation of the zig-zag from Figure 3 by a blind turtle with two counters. Symbols $m / n$ indicate step $m$ along vertical line $n$.

in addition to the finite-state control. There is no upper limit on the magnitude of numbers that can be represented by either counter, thus their inclusion implies infinite memory. Counter $m$ indicates the number of line segments that have already been created along the vertical line that is being drawn. Counter $n$ indicates the target length of that vertical line, which is equal to its ordering number, counting from the left side of the pattern. When $m=n$, the turtle performs a U-turn to the left or right. The value of $m$ is then set to 0 and the value of $n$ is incremented by 1 , which initializes the drawing of the next vertical line.

How realistic is this algorithm as a possible description of a real-world process? We can imagine that it could be used by a blindfolded person who was given the task of tracing the zig-zag curve of Figure 3, although the maintenance of straight lines and turning angles of proper magnitudes would not be easy. It is more difficult to imagine an agent with infinite memory in the context of a natural morphogenetic process, but counting capabilities have been postulated to explain the formation of plants that produce a constant number of leaves before they start flowering, for example in ${ }^{31}$.

The blind agent with counters is a special case of a blind agent with infinite memory. We can expect that agents with different computational capabilities may lead to different classes of generated patterns. A study of these relationships may link, in an interesting manner, problems of pattern formation to the theory of automata and formal languages. Previous work along these lines has been reported by Maurer et al., ${ }^{19}$ and Sudborough and Welzl. ${ }^{39}$

In many cases, the assumption of infinite memory is unrealistically strong. In the following sections we will examine several ways in which it can be avoided.

\section{Single sighted agent receiving cues from the environment (one-way exogenous control)}

The computational capacities of the turtle can be reduced if the turtle is allowed to receive cues from its environment. In the example shown in Figure 7 it is assumed that the environment is a wedge limited by two intersecting guiding lines. The turtle moves vertically until it runs into any of these guiding lines, at which time it performs a U-turn.

This process can be related to the formation of zig-zag patterns by leaf miners, insect larvae that live and feed within a leaf. In the process of feeding, they create 

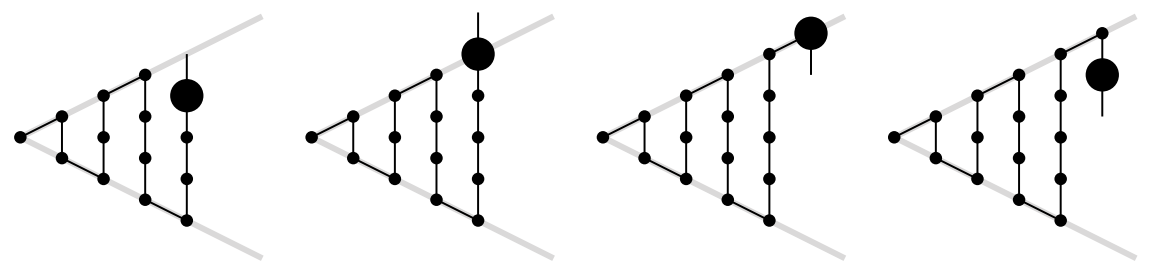

Figure 7: Zig-zag created by a turtle receiving cues from the environment

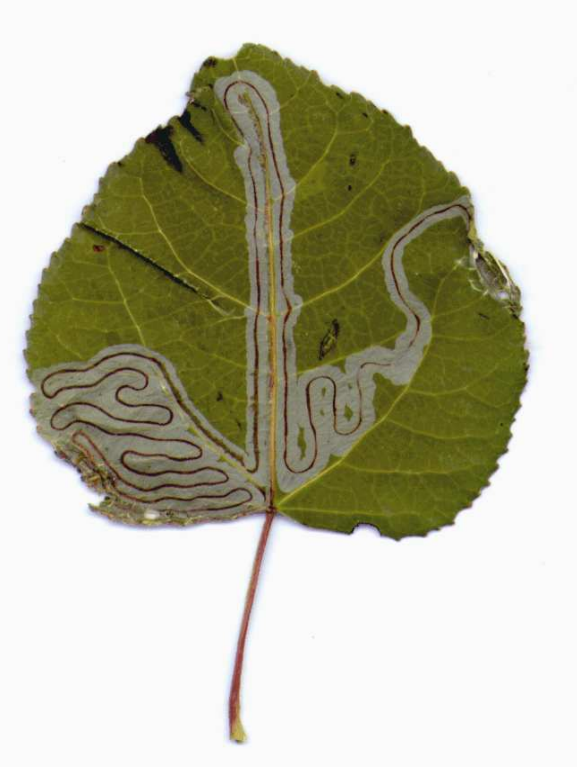

Figure 8: A leaf with a pattern of tunnels created by a leaf miner

tunnels that are visible as thin trails in the leaf blade. An example of the resulting pattern is shown in Figure 8. It has a meandering character reminiscent of a zig-zag curve, and is limited by the major leaf veins, which the larva cannot easily cross.

From a more general perspective, the guiding lines in Figure 7 provide the turtle with the information about its position within its environment. Positional and directional cues have a fundamental impact on animal behavior, ranging from the maintenance of proper distance from the shore by many littoral organisms to the orientation of insects and birds with respect to the sun, and the use of landmarks by animals that are heading towards a familiar goal. ${ }^{4}$ Although these behaviors often do not leave visible traces, they nevertheless can be conceptualized as paths in space, and interpreted as patterns.

The use of environmental cues is also a ubiquitous factor in plant development. For example, the development of climbing plants is guided by the geometry of their support. ${ }^{28}$ 

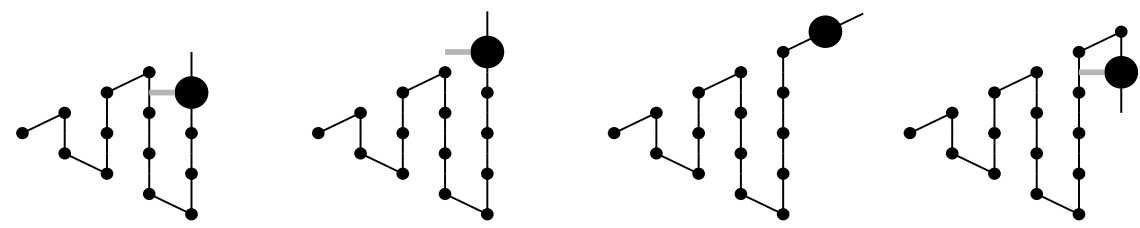

Figure 9: Zig-zag created by a turtle receiving cues from the structure (a-d)

At a lower level of organism development, the assumption that morphogenetic agents have access to positional information is the cornerstone of the pattern formation theory proposed in 1970 by Wolpert. ${ }^{47}$ He suggested that a cell in a developing organism may "know" its position with respect to one of more reference points, and may use this information to determine the nature of its differentiation.

Since position with respect to a set of reference points is a global attribute of a cell, the use of positional information may seem inconsistent with the goal of describing pattern formation as a local-to-global process. This apparent inconsistency can be solved by considering morphogenesis as a two-level phenomenon. At the higher level, a field of values representing positional information is established by a local mechanism, for example, as a field of concentrations of a substance that diffuses from a source to a sink. At the lower level, each cell accesses the local field value associated with its position, and interprets this information as a factor guiding its development. ${ }^{47}$

\section{Single sighted agent receiving cues from the structure (two-way ex- ogenous control)}

The mechanism of positional control considered in the previous section operated under the assumption of one-way information flow from the environment to the agent. If we allow the turtle not only to passively obtain environmental cues, but also to change its environment, the use of global positional information may become unnecessary.

To illustrate this, let us consider the generation of the zig-zag pattern shown in Figure 9. In this case, the pattern becomes a part of the environment as it is created. The turtle can perceive this environment within some finite neighborhood around itself. To form the zig-zag pattern, the turtle walks next to the previously created vertical line, sensing its presence in each step (Figure 9a). When the turtle detects that it has walked past the previous line (b), it turns around (c) and resumes the creation of the next vertical line (d).

Cues from the structure created so far may have been used already by the leaf miner to keep an approximately constant distance between adjacent parts of the tunnel, and prevent it from self-intersecting. Similar patterns, known as trace fossils, have been created by early multicellular organisms (over 500 million years ago), and have been preserved in the fossil record. As described by Prescott and Ibbotson, ${ }^{25}$ "the earliest traces reflect simple 'scribbling' behaviors, with tracks that often cross themselves and indicate relatively crude foraging strategies. [Later], however, more 

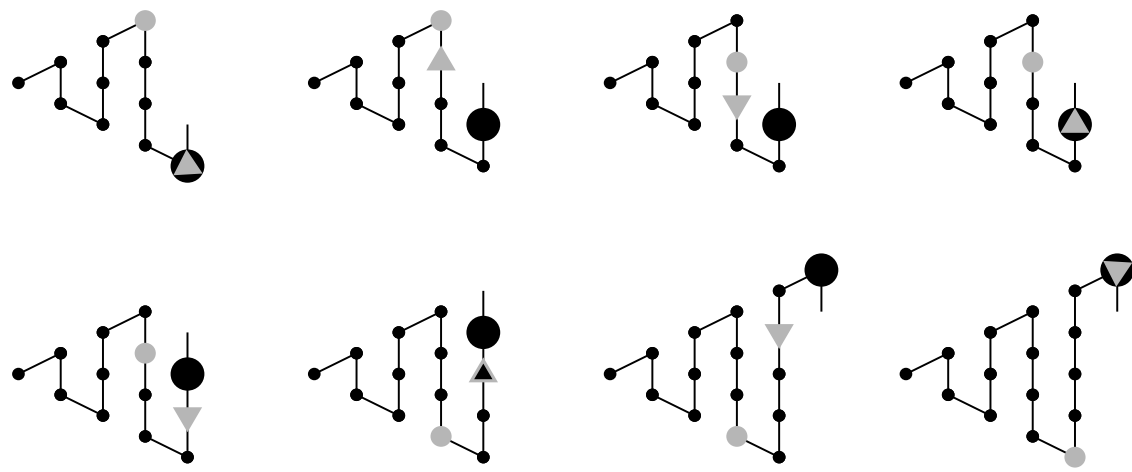

Figure 10: Construction of the zig-zag curve using endogenous information flow $(\mathrm{a}-\mathrm{h})$

regular trails appear that form spirals or 'meanders' that loop back on themselves without crossing." Computer simulations of these patterns were presented as early as 1969 by Raup and Seilacher. ${ }^{33,34}$ More recently, Prescott and Ibbotson recreated similar patterns using robots laying a paper trace on the floor, and sensitive to the track created so far. ${ }^{25}$

Construction of orb webs by spiders is another example of pattern formation in which the agent receives cues from the structure being constructed. As described by Krink and Vollrath, ${ }^{12}$ for example, an orb spider begins web construction by creating an outer frame and a set of radii that run between the hub near the web center and the frame. It then lays typically two spirals: an auxiliary spiral (from the inside out) and the capture spiral (from the outside in). When constructing the spirals, the spider uses the previously laid coil as a reference for measuring distance to the next coil.

\section{Single self-controlled agent (endogenous control)}

In the case of exogenous control, the agent can receive information about the structure completed so far through the space in which this structure is embedded. As noted by Bell ${ }^{3}$ (see Section 1), another important possibility is the transfer of information through the structure itself. We will use the term "endogenous information transfer" as a synonym for the term "self-control" to denote this type of pattern-formation mechanism. ${ }^{26}$

Construction of the zig-zag pattern using endogenous information flow is illustrated in Figure 10. As in the case of exogenous control (Figure 9), the turtle uses the last completed vertical line to guide the creation of the next line. Now, however, all information propagates through the zig-zag curve. Specifically, the turtle uses signals (grey triangles in Figure 10) to communicate with a pebble (grey circle), which can be placed at any node between two consecutive line segments. When the creation of a new vertical line begins, the pebble is located at the far end of the previous line (Figure 10a). The turtle then moves forward, drawing a line segment, and sends signal $A$ to the pebble (b). Upon the receipt of this signal, the pebble 
moves to the next node and sends a return signal $B$ to the turtle (c). When this signal is received (d), the turtle moves forward, creating the next line segment, and resends signal $A$ to the pebble (e). This initiates another sequence of steps similar to that depicted in figures (b,c,d). The back-and-forth exchange of signals between the turtle and the pebble synchronizes the addition of line segments by the turtle with the movements of the pebble from one node to the next. Thus, the previous line is used as a template for creating the next line. This cycle is modified when the pebble reaches a corner. In that case, a different signal $B^{\prime}$ is sent to the turtle (f), instructing it to create an additional vertical line segment followed by a diagonal line $(\mathrm{g})$. This modified cycle is responsible for adding an extra segment to the current line, making it one segment longer than its predecessor. Afterwards, the pebble is moved to the beginning of the next (just completed) vertical line, and the turtle is ready to draw the first segment of the new vertical line $(\mathrm{h})$. The state of the system is now similar to that depicted in Figure (a), and the extension of the zig-zag curve with the subsequent line segments will proceed in a manner analogous to that already described.

Taken literally, this example may seem contrived: it is hard to imagine a morphogenetic process that would use signals and a pebble in a similar way. Nevertheless, the proposed mechanism is related to self-reproduction, an attribute of life that has been extensively studied from a formal perspective. ${ }^{13,14,46}$ Mathematically, selfreproduction is a process in which a structure embedded in a homogeneous space (originally defined in terms of cellular automata) creates a potentially infinite number of copies of itself. The described method for generating the zig-zag curve uses the same general paradigm: it creates new vertical lines by copying — with the addition of an extra line segment - the previously constructed lines.

Many physiological mechanisms, such as propagation of nutrients, water, and hormones in plants can also be conceptualized as endogenous information flow. We will consider models of processes that use endogenous information flow in Sections 10 and 11.

\section{Multiple blind agents with finite memory}

In Sections 2 to 6 we have shown that a finite-state turtle can generate a zig-zag curve of increasing amplitude if it receives appropriate cues from the environment, or if it can inspect the structure constructed so far. The next example illustrates another possibility. A growing set of agents, acting in concert, can create the zig-zag curve even if each agent has only finite memory and acts autonomously, without any input.

Recall that an autonomous finite-state turtle can create a zig-zag line of constant amplitude (Figure 4). A modified version of that pattern, with slanted line segments instead of horizontal lines, is shown in Figure 11 (top). Let us now assume that the turtle spawns auxiliary agents, represented as grey squares, and deposits them in obtuse-angled corners of the curve. If these agents act in synchrony with the turtle, and each agent extends its associated vertical line once per every two turtle steps, a zig-zag curve of increasing amplitude will result (Figure 11, bottom). Neither the turtle nor the auxiliary agents receive any information from the environment: 

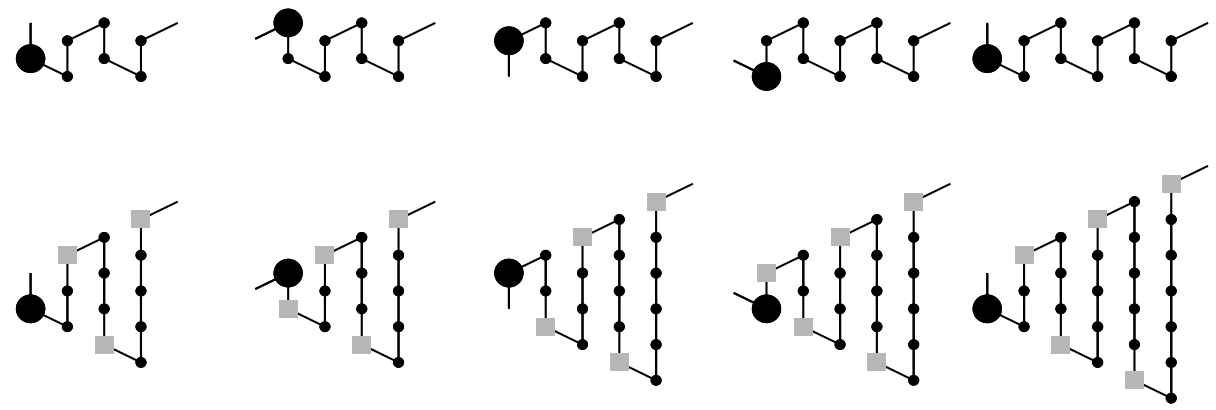

Figure 11: Zig-zag created by multiple autonomous agents with finite-state control
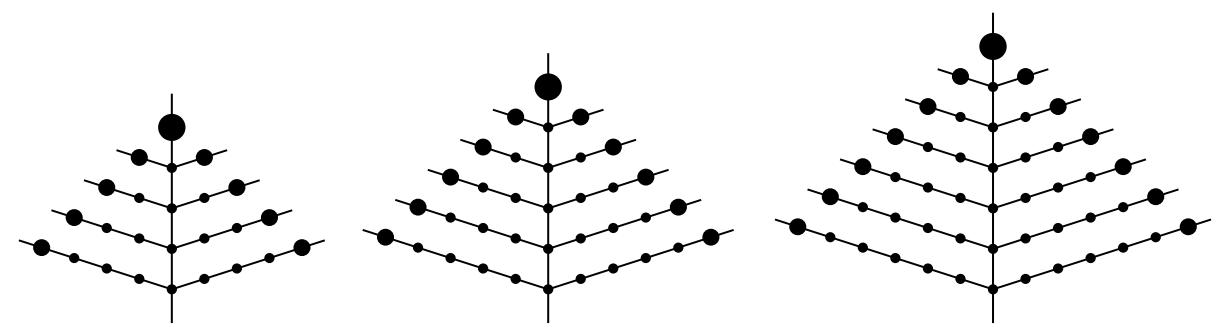

Figure 12: Development of a basitonic branching structure

each of them acts autonomously, cycling through a predefined sequence of steps and actions. The increasing zig-zag amplitude is a consequence of the sequential initiation of vertical lines by the turtle. Since the agents extend all vertical lines synchronously, the lines created earlier have more time to grow and therefore will become comparatively longer.

This mechanism can be thought of as a prototype of the development of many plants. The agents correspond to apical meristems that contribute new line segments - the internodes - to a branching structure, and create new meristems. A sample structure produced by such a process is shown in Figure 12. The main meristem creates a sequence of internodes along the vertical axis of the model. Each of these internodes is associated with a lateral meristem which, in turn, produces a sequence of internodes that become a lateral branch. The operation of all meristems is synchronized. Due to the sequential creation of the lateral apices, the branches at the bottom of the structure have more time to grow than those at the top, and the generated structure has a characteristic, basitonic shape: it is more developed at the base than at the top.

The development of branching structures by populations of autonomous agents with finite memory can be conveniently expressed using the formalism of L-systems without interactions (0L-systems). ${ }^{16}$ This class of L-systems has been extensively studied $^{35}$ and applied to the synthesis of many realistic plant models. ${ }^{31,32}$ 

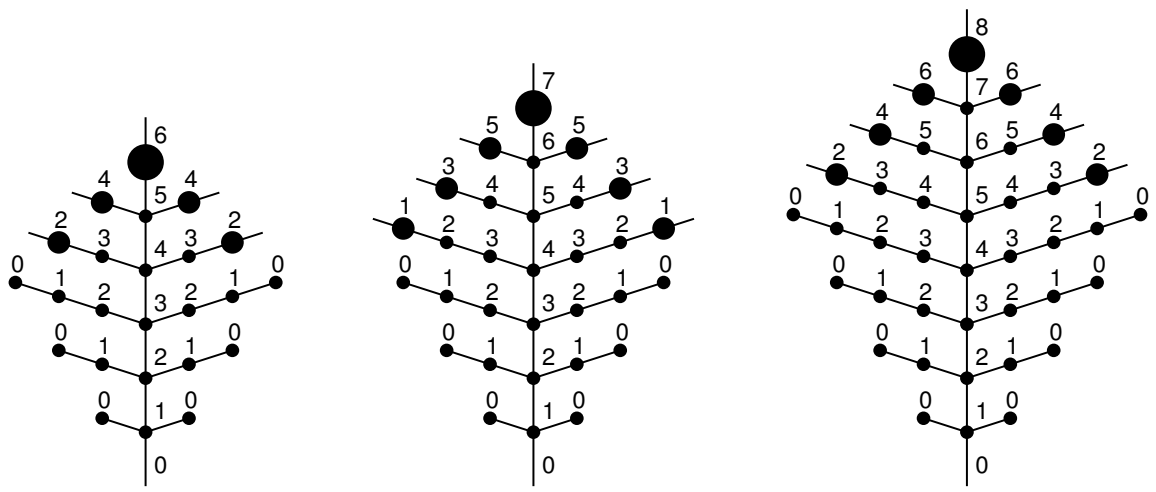

Figure 13: Construction of a mesotonic branching pattern by a growing set of turtles with counting capabilities. Numbers indicate the states of the turtles at their current and past positions.

\section{Multiple blind agents with infinite memory}

In nature, one observes not only basitonic, but also mesotonic and acrotonic structures, characterized by extensive development of the middle or upper parts of the plant, respectively. These structures are counterintuitive in the sense that higher branches, although produced by lateral apices that have been created more recently than the bottom ones, are nevertheless longer then the lower branches. It has been shown that (potentially infinite) mesotonic and acrotonic structures cannot be generated by a population of autonomous meristems with finite memory, located at the apices of the axes as observed in real plants. ${ }^{5,30}$ Consequently, several more powerful mechanisms have been proposed in the literature. Specifically, Lück et al. ${ }^{17}$ described a model in which the meristems can count. As shown in Figure 13, the apex of the main axis counts the segments that it has created so far, and assigns the resulting values to the counters associated with the newly created lateral apices. These apices count down as they create new segments, and stop at zero. Consequently, the length of each fully developed lateral axis is equal to its position on the main axis (measured in the number of internodes), yielding a mesotonic structure. (It is not acrotonic because of the juvenile axes of decreasing length at the top.) Biologically, the counter values might be represented by concentrations of substances, which accumulate in the apices and can be dissipated by them.

In the above example, each agent has only one counter. In contrast, the autonomous turtle with infinite memory that created the zig-zag pattern described in Section 3 (Figure 6) required the use of two counters. Apparently, the addition of one counter does not increase the computing power of a single autonomous agent with finite memory, but increases the power of a set of such agents.

\section{Multiple sighted agents}

In the case of curves created by a single agent, we have observed a tradeoff between memory size and the input information available to the agent. For example, 


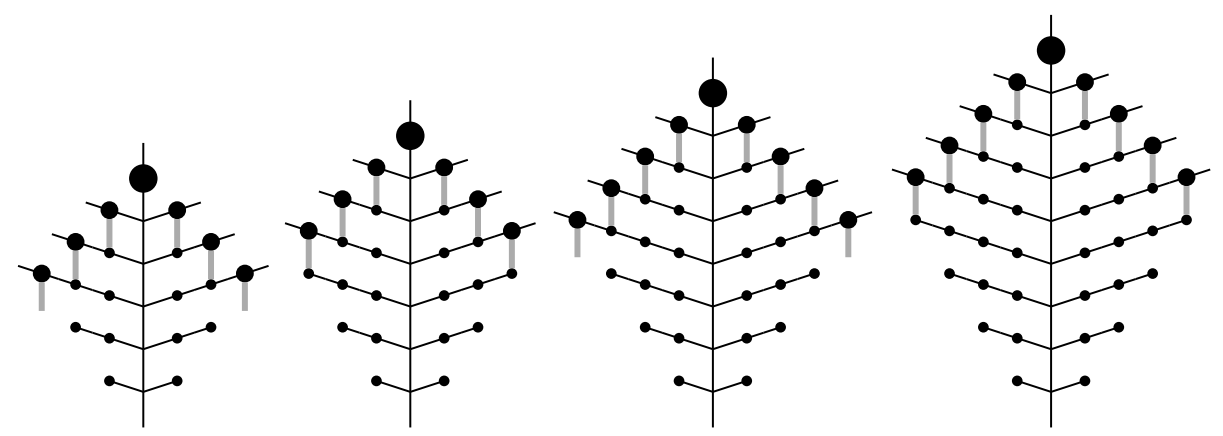

Figure 14: Construction of a mesotonic branching pattern by multiple sighted agents
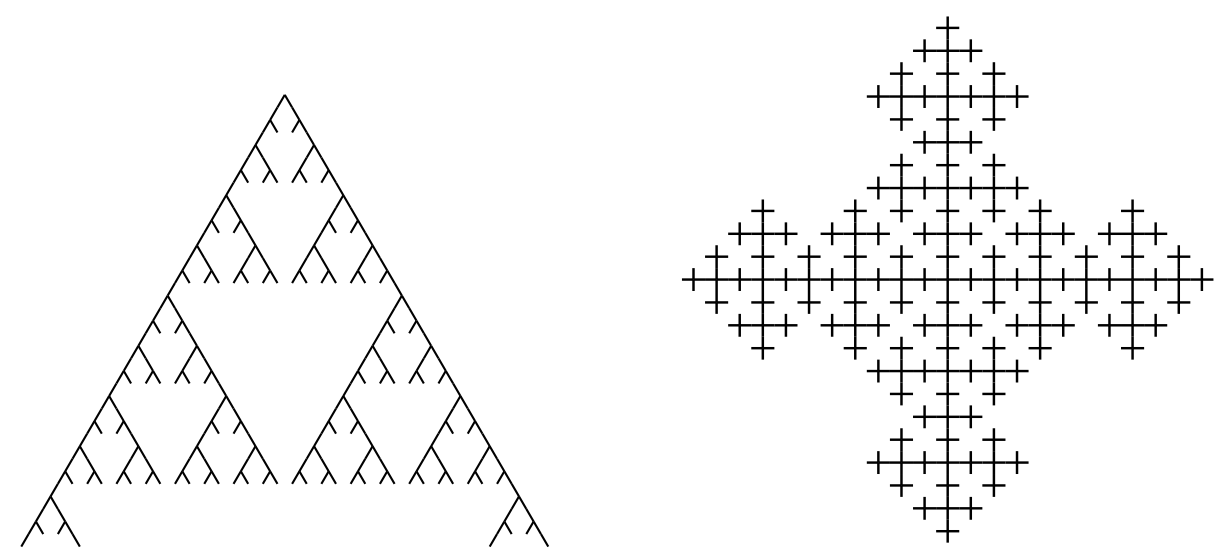

Figure 15: Examples of patterns created by multiple sighted turtles that stop the creation of new branches to prevent collisions: Sierpiński gasket ${ }^{36}$ (left) and Ulam's Maltese crosses ${ }^{44,45}$ (right)

the exogenously-controlled turtle, which perceived its environment within some finite neighborhood, no longer needed infinite memory to create the zig-zag curve (Section 5 and Figure 9). The development of branching structures by multiple agents is subject to similar tradeoffs. Specifically, a mesotonic branching structure can be created by a collection of exogenously-controlled turtles with finite memory (Figure 14). To accomplish this, the turtle creating branch $n+1$ follows branch $n$ immediately below it, and adds one extra segment when the end of that branch has been reached. Branch $n+1$ will then have one more segment than branch $n$.

The collective behavior of a growing set of sighted finite-memory agents is often much less obvious than in the previous example. For instance, Figure 15 shows two patterns created by sets of agents that create new branches (and new agents) only if this does not lead to collisions. In both cases, the fractal nature of the resulting patterns would be difficult to predict. 


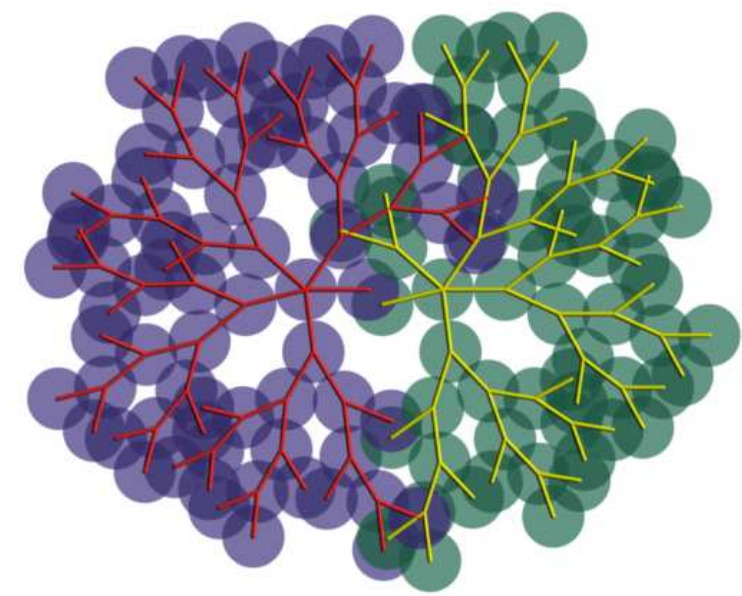

Figure 16: A top view of a branch tier created according to the model by Honda et al. ${ }^{11}$. The apices stop growing if they run into clusters of leaves located in the nodes of the structure or associated with other apices.

Plants use forms of collision-detection mechanisms to prevent excessive proliferation of branches. For example, Figure 16 shows a model of a branch tier of the tropical tree Terminalia catappa. This model was originally proposed by Honda et $a l .{ }^{11}$ The development of a branch is terminated when its apical meristem runs into a leaf cluster at any of the existing or newly formed nodes of the structure. For a detailed description of this model see ${ }^{11,22}$.

Honda's model does not simulate the physical or physiological processes through which a collision between an apex and leaf cluster might be detected, and only captures the effect of this interaction. In nature, the proximity of other branches is oftern sensed as a change in the intensity and quality of light (red / far red ratio) reaching a meristem. The meristem may respond by slowing down the production of new internodes and lateral apices, and eventually terminating the growth of an axis altogether. For example, Figure 17 shows the developmental model of two clonal plants (Portulaca oleracea L.), based on a qualitative description the neighborsensing mechanism presented by Novoplansky et al. ${ }^{23}$ Proximity of other branches is perceived as a local change in the red / far red ratio of light distributed in the canopy. Light distribution was simulated using the Monte Carlo method. ${ }^{21}$

\section{Multiple endogenously-controlled agents}

As evident in the simulations of zig-zag patterns discussed earlier, exogenous and endogenous control mechanisms may yield similar structures, although the internal mechanism of their operation is different. Correspondingly, a mesotonic branching structure can also be created by a growing population of apices that communicate 


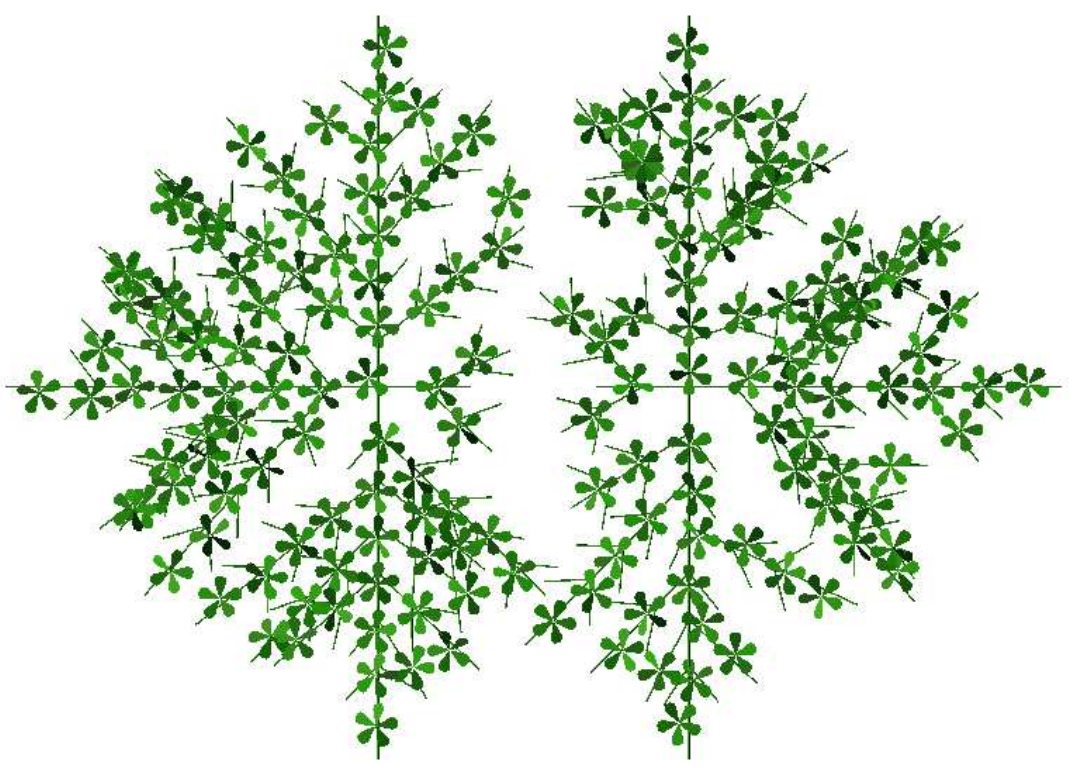

Figure 17: A model of two portulaca plants, growing close together. The apices detect the proximity of parts of the same or another plant, and use this information to avoid collisions. In reality and in the model, the neighbor-detection mechanism is based on sensing the quality of light (red / far red ratio), which is changed by reflection and transmission of light through the leaves.

endogenously. An example is given in Figure 18. The apex of the main axis produces a sequence of vertical internodes and lateral apices as in Figures 13 and 14. These apices create lateral branches by periodically adding new internodes to them. This process is controlled by signals that propagate from the base of the plant towards the apices (grey triangles in Figure 18). When a signal reaches the apex of a lateral
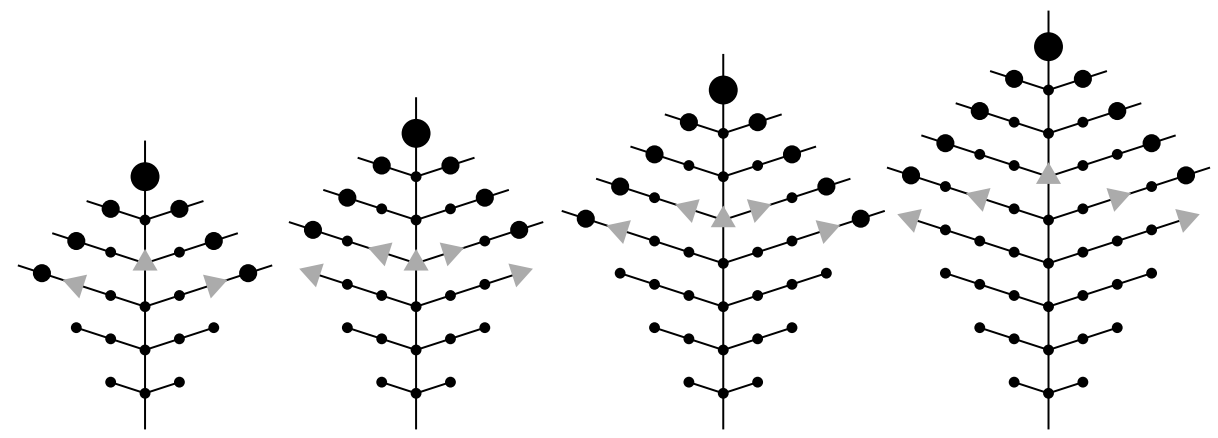

Figure 18: Development of a mesotonic branching pattern, with the branch lengths controlled by signals (endogenously) 


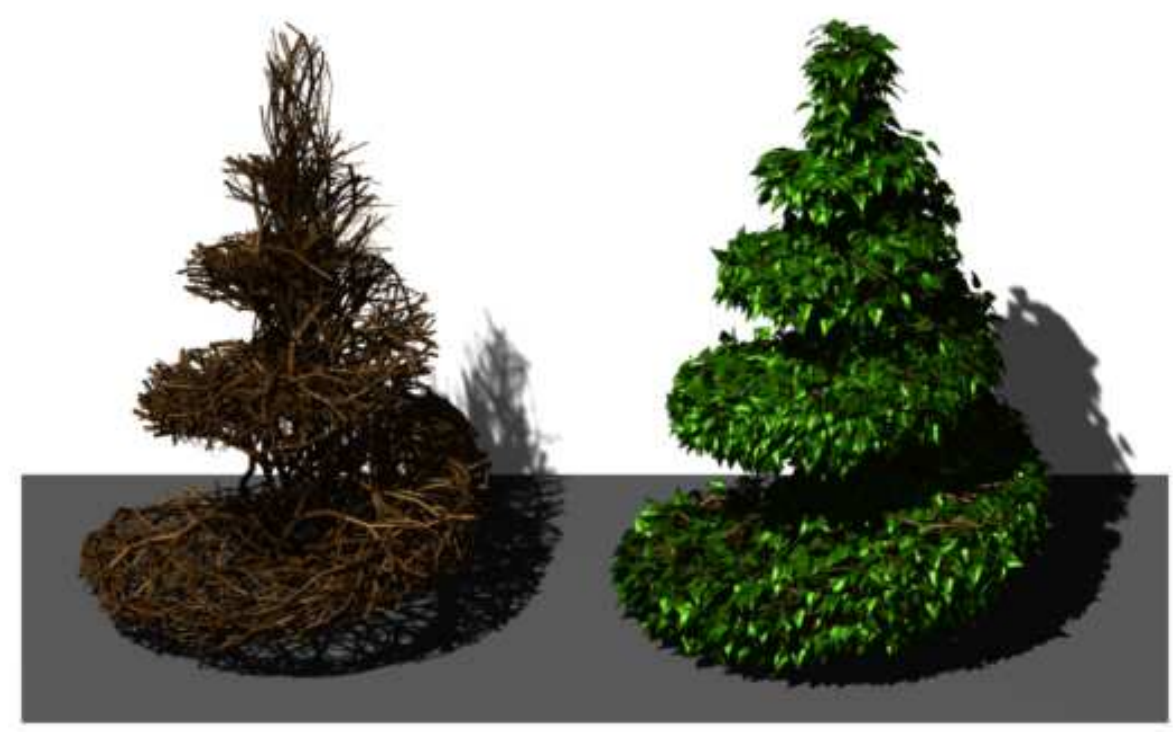

Figure 19: Simulated trees pruned to a spiral shape. From ${ }^{29}$.

branch, it terminates its growth. If proper signal propagation rates along the main axis and in lateral branches are maintained, each successive branch is allowed to grow for a longer time than its predecessor, resulting in a gradual increase of the branch length. For a detailed description of this model see ${ }^{28}$.

\section{Combined effect of multiple mechanisms}

Analysis of individual pattern formation mechanisms is important from the theoretical point of view, since it illuminates the generative capacity of each mechanism, and the relationships between them. In nature, different mechanisms often operate in concert. The pruning of trees is one example. In this case, a plant receives cues from its environment (location of cut points on selected branches), and translates them into endogenous signals. The signals activate dormant buds, prompting their growth into new branches near the pruning sites. By repetitive pruning and regrowth, plants can be sculpted into artificial forms (topiary). A topiary tree model that simulates these processes is shown in Figure 19. A detailed description of this model is presented in ${ }^{29}$.

Another multi-mechanism model, proposed by Takenaka ${ }^{40}\left(\right.$ see also $\left.^{22}\right)$, explains the branching pattern and overall shape of trees that grow in isolation or in stands. Light reaching leaves (an exogenous factor) causes production of photosynthates. These photosynthates are used locally to maintain current structure and create new branches or branch segments. The remainder is transported towards branch bases (an endogenous mechanism). If the amount of photosynthates available for 


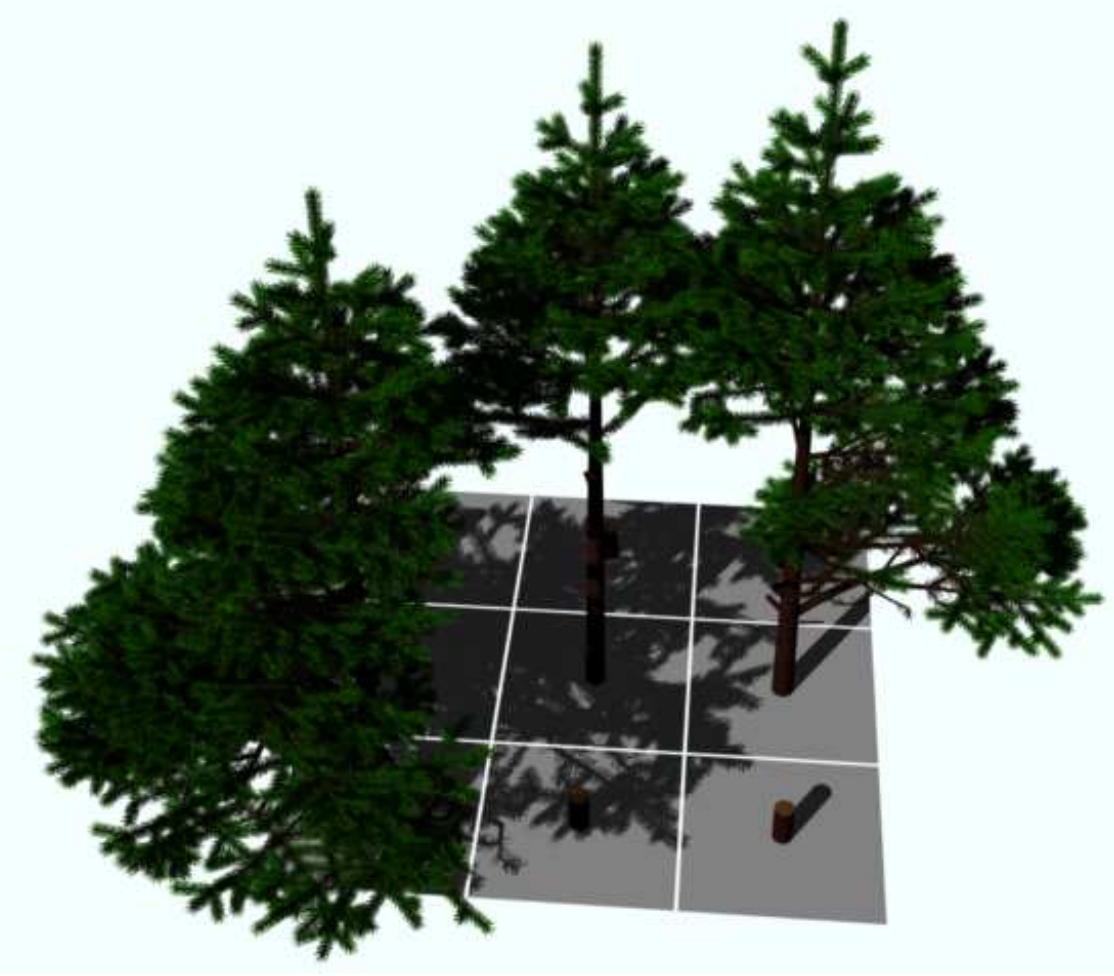

Figure 20: Simulated relationships between tree form and its position in the stand. The model integrates exogenous and endogenous control mechanisms. From ${ }^{22}$.

the production of new branches is too small, the apex will add new segments to the current axis without initiating new branches, or will terminate the growth of the current axis altogether. Furthermore, if the amount of photosynthates reaching the base of an entire branch is less than the amount needed to maintain it, the branch will become a liability and will be shed by the tree. A simulated model of a tree stand, with each tree employing the above mechanisms, is shown in Figure 20. The global shape of the crowns is an emergent property of local, exogenous and endogenous control mechanisms incorporate in the model. For details see ${ }^{22}$.

Comprehensive models of plant structures that integrate various developmental mechanisms have been termed functional-structural models. ${ }^{37}$ They are being studied with agriculture, forestry, and horticulture applications in mind. ${ }^{27}$ This shows that studies of pattern formation are not only interesting from a theoretical perspective, but may also lead to practical applications.

\section{Conclusions}

In this paper we considered the generation of simple patterns - a zig-zag curve and a mesotonic branching pattern - as a vehicle for illustrating different algorithms of 
pattern formation. The relevance of these algorithms was supported by examples of their occurrence in nature. The description of the algorithms was guided by three independent criteria: the number of agents (one or many), the computing capability of each agent (a finite automaton or an automaton with counters), and its capability of exchanging information with the environment (one-way exogenous, two-way exogenous, or endogenous information flow).

Some obvious cases were not included in the discussion - for example, patterns created by a constant number of agents greater than one, patterns created by multiple asynchronous agents, and self-assembling patterns created by agents that move freely in space and connect themselves according to locally specified criteria. Furthermore, the presentation of different pattern formation mechanisms was carried out at the intuitive level, without formal definitions and proofs. Nevertheless, it seems possible to complete this classification, formulate the theorems, and present their proofs using the main lines of thought outlined in this paper. The resulting theory would formally characterize the tradeoffs between the informationprocessing capabilities of individual agents, their forms of communication, and their individual or concerted operation. It is possible that these tradeoffs would echo the relationships between different complexity measures studied in the theory of algorithms, effectively extending it to patterns and pattern formation processes. This could provide a firm basis for hypothesizing about the most plausible mechanisms responsible for the formation of specific patterns in nature using the parsimony argument: the most likely explanation is the simplest one. The creation of a computational theory of pattern formation seems a fascinating and rewarding task.

\section{Acknowledgments}

I would like to thank Radomír Měch for his work on several images included in this paper, Martin de Boer for the leaf with the miner pattern, Shane Dorosh for surveying the literature on the behavior and modeling of spiders, and Lynn Mercer for helpful editorial comments. This work has been supported in part by research and equipment grants from the Natural Sciences and Engineering Research Council of Canada.

\section{References}

1. H. Abelson and A. A. diSessa. Turtle geometry. M.I.T. Press, Cambridge, 1982 .

2. A. Bell. Dynamic morphology: a contribution to plant population ecology. In R. Dirzo and J. Sarukhán, editors, Perspectives on plant population ecology, pages 48-65. Sinauer, Sunderland, MA, 1984.

3. A. D. Bell. The simulation of branching patterns in modular organisms. Philos. Trans. Royal Society London, Ser. B, 313:143-169, 1986.

4. F. C. Dyer. Cognitive ecology of navigation. In R. Dukas, editor, Cognitive ecology. The evolutionary ecology of information processing and decision making, pages 201-260. University of Chicago Press, Chicago, 1998. 
5. D. Frijters and A. Lindenmayer. Developmental descriptions of branching patterns with paracladial relationships. In A. Lindenmayer and G. Rozenberg, editors, Automata, languages, development, pages 57-73. North-Holland, Amsterdam, 1976.

6. B. Grünbaum. Periodic ornamentation of the fabric plane: lessons from Peruvian fabrics. Symmetry, 1(1):45-68, 1990.

7. B. Grünbaum and G. C. Shephard. Tilings and patterns. W. H. Freeman and Company, New York, 1987.

8. L. I. Held. Models for embryonic periodicity, volume 24 of Monographs in Developmental Biology. Karger, Basel, 1994.

9. S. Hildebrandt and A. Tromba. The parsimonious universe: Shape and form in the natural world. Copernicus (Springer), New York, 1996.

10. B. Hölldobler and M. Möglich. The foraging system of Pheidole militicida (Hymenoptera: Formicidae). Insectes Sociaux, 27(3):237-264, 1980.

11. H. Honda, P. B. Tomlinson, and J. B. Fisher. Computer simulation of branch interaction and regulation by unequal flow rates in botanical trees. American Journal of Botany, 68:569-585, 1981.

12. T. Krink and F. Vollrath. Analysing spider web-building behaviour with rulebased simulations and genetic algorithms. Journal of Theoretical Biology, 185:321-331, 1997.

13. R. Laing. Automaton models of reproduction by self-inspection. Journal of Theoretical Biology, 66:437-457, 1977.

14. C. Langton. Self-reproduction in cellular automata. Physica D, 10:135-144, 1984.

15. A. Lindenmayer. Mathematical models for cellular interaction in development, Parts I and II. Journal of Theoretical Biology, 18:280-315, 1968.

16. A. Lindenmayer. Developmental systems without cellular interaction, their languages and grammars. Journal of Theoretical Biology, 30:455-484, 1971.

17. J. Lück, H. B. Lück, and M. Bakkali. A comprehensive model for acrotonic, mesotonic, and basitonic branching in plants. Acta Biotheoretica, 38:257-288, 1990.

18. G. E. Martin. Transformation geometry. An introduction to symmetry. Springer-Verlag, New York, 1982.

19. H. A. Maurer, G. Rozenberg, and E. Welzl. Using string languages to describe picture languages. Information and Control, 54:155-185, 1982.

20. H. Meinhardt. The algorithmic beauty of sea shells. Springer-Verlag, Berlin, 1995.

21. R. Měch. Modeling and simulation of the interactions of plants with the environment using L-systems and their extensions. $\mathrm{PhD}$ thesis, University of Calgary, October 1997.

22. R. Měch and P. Prusinkiewicz. Visual models of plants interacting with their environment. Proceedings of SIGGRAPH '96 (New Orleans, Louisiana, August 4-9, 1996) ACM SIGGRAPH, New York, 1996, pp. 397-410.

23. A. Novoplansky, D. Cohen, and T. Sachs. How portulaca seedlings avoid their neighbours. Oecologia, 82:490-493, 1990. 
24. G. Pólya. Über die Analogie der Kristallsymmetrie in der Ebene. Z. Krist., 60:278-282, 1924.

25. T. J. Prescott and C. Ibbotson. A robot trace maker: modeling the fossil evidence of early invertebrate behavior. Artificial Life, Vol 3:289-306, 1997.

26. P. Prusinkiewicz. Visual models of morphogenesis. Artificial Life, 1(1/2):6174, 1994.

27. P. Prusinkiewicz. Modeling of spatial structure and development of plants: a review. Scientia Horticulturae, (74):113-149, 1998.

28. P. Prusinkiewicz, M. Hammel, J. Hanan, and R. Měch. Visual models of plant development. In G. Rozenberg and A. Salomaa, editors, Handbook of formal languages, Vol. III: Beyond words, pages 535-597. Springer, Berlin, 1997.

29. P. Prusinkiewicz, M. James, and R. Měch. Synthetic topiary. Proceedings of SIGGRAPH '94 (Orlando, Florida, July 24-29, 1994). ACM SIGGRAPH, New York, 1994, pp. 351-358.

30. P. Prusinkiewicz and L. Kari. Subapical bracketed L-systems. In J. Cuny, H. Ehrig, G. Engels, and G. Rozenberg, editors, Graph grammars and their application to computer science; Fifth International Workshop, Lecture Notes in Computer Science 1073, pages 550-564. Springer-Verlag, Berlin, 1996.

31. P. Prusinkiewicz and A. Lindenmayer. The algorithmic beauty of plants. Springer-Verlag, New York, 1990. With J. S. Hanan, F. D. Fracchia, D. R. Fowler, M. J. M. de Boer, and L. Mercer.

32. P. Prusinkiewicz, A. Lindenmayer, and J. Hanan. Developmental models of herbaceous plants for computer imagery purposes. Proceedings of SIGGRAPH '88 (Atlanta, Georgia, August 1-5, 1988). In Computer Graphics 22, 4 (August 1988), pages 141-150, ACM SIGGRAPH, New York, 1988.

33. D. M. Raup. Modeling and simulation of morphology by computer. In Proceedings of the North American Paleontology Convention, pages 71-83, 1969.

34. D. M. Raup and A. Silacher. Computer simulation of fossil foragin behavior. Science, 166:994-995, 1969.

35. G. Rozenberg and A. Salomaa. The mathematical theory of L systems. Academic Press, New York, 1980.

36. W. Sierpiński. Sur une courbe dont tout point est un point de ramification. Comptes Rendus hebdomadaires des séances de l'Académie des Sciences, 160:302-305, 1915. Reprinted in W. Sierpiński, Oeuvres choisies, S. Hartman et al., editors, pages 99-106, PWN - Éditions Scientifiques de Pologne, Warsaw, 1975.

37. R. Sievänen, A. Mäkelä, E. Nikinmaa, and E. Korpilahti. Preface. Silva Fennica, 31(3):237-238, 1997. Special issue on functional-structural tree models.

38. P. S. Stevens. Patterns in nature. Little, Brown and Co., Boston, 1974.

39. I. H. Sudborough and E. Welzl. Complexity and decidability for chain code picture languages. Theoretical Computer Science, 36:173-202, 1985.

40. A. Takenaka. A simulation model of tree architecture development based on growth response to local light environment. Journal of Plant Research, 107:321-330, 1994.

41. d'Arcy Thompson. On growth and form. University Press, Cambridge, 1952. 
42. T. Toffoli and N. Margolus. Cellular automata machines: A new environment for modeling. MIT Press, Cambridge, Massachusetts, 1987.

43. A. Turing. The chemical basis of morphogenesis. Philosophical Transactions of the Royal Society of London B, 237:37-72, 1952.

44. S. Ulam. On some mathematical properties connected with patterns of growth of figures. In Proceedings of Symposia on Applied Mathematics, volume 14, pages 215-224. American Mathematical Society, 1962.

45. S. Ulam. Patterns of growth of figures: Mathematical aspects. In G. Kepes, editor, Module, proportion, symmetry, rhythm, pages 64-74. Braziller, New York, 1966.

46. J. von Neumann. Theory of self-reproducing automata. University of Illinois Press, Urbana, 1966. Edited by A. W. Burks.

47. L. Wolpert. Positional information and pattern formation. In C. H. Waddington, editor, Towards a theoretical biology, Vol. 3, pages 198-230. Edinburgh University Press, Edinburgh, 1970. 